Onkologie 1996;19:I-X

\title{
Inhalt, Vol. 19, 1996
}

\section{Schriftleitung}

W. Queißer, Mannheim H. Huber, Wien

Fachschriftleitung

W. E. Berdel, Berlin E. Dühmke, München R. Hartenstein, München M. Kaufmann,

Frankfurt/M. H. Rübben, Essen P. Schlag, Berlin

Wissenschaftlicher Beirat

L. Bergmann, Frankfurt/M.

C. Bokemeyer, Tubingen

U. Creutzig, Münster

V. Diehl, Köln

P. Drings, Heidelberg

L. Edler, Heidelberg

G. Eisenbrand, Kaiserslautern

H. H. Fiebig, Freiburg

H. Gadner, Wien

K. Havemann, Marburg

F. Herrmann, Ulm

R. Herrmann, Basel

D. Hoelzer, Frankfurt/M.

H. J. Illiger, Oldenburg

R. Jakesz, Wien

F. Jänicke, Hamburg

U. R. Kleeberg, Hamburg

R. Kreienberg, Ulm

M. Lehnert, St. Gallen

H. Löffler, Kiel

H. Ludwig, Wien

Chr. Mannhalter, Wien

H. J. Meyer, Hannover FA. Muthny, Münster G. A. Nagel, Freiburg M. Neises, Mannheim R. Parwaresch, Kiel

A. Pfleiderer, Freiburg

M. Pfreundschuh, Homburg/Saar

F. Porzsolt, Ulm

K. Possinger, Berlin H. Samonigg, Graz H. Sauer, München

G. Schackert, Dresden

W Scheithauer, Wien

H.-J. Schmoll, Halle

R. Schulte-Hermann, Wien S. Seeber, Essen W Siegert, Berlin 
B. Thürlimann, St. Gallen

W. Tilgen, Heidelberg

M. Wannenmacher, Heidelberg H. J. Weh, Hamburg Th. Wendt, Jena H. Wilke, Essen M. Wirth, esdenKAIUiER

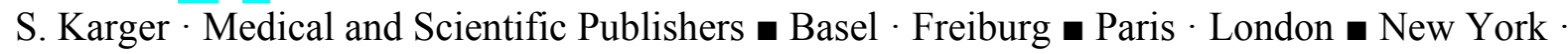
New Delhi $\cdot$ Bangkok $\cdot$ Singapore $\cdot$ Tokyo $\cdot$ Sydney

Die Zeitschrift erscheint zweimonatlich; pro Jahr er-scheint 1 Band zu je 6 Heften. Bezugspreís für Jahrgang 19, 1996, DM 182-/SFr 142,-. einschließlich MwSt., zuzüg-lich Postgebühren. Der Abonnementpreis ist im voraus zahlbar. Das Abonnement der Zeitschrift läuft weiter, wenn es nicht spätestens 4 Wochen vor Abschluß eines Bandes abbestellt wird.

Abonnementbestellungen können bei jeder Buchhand-lung oder direkt beim Verlag aufgegeben werden: Bundesrepublik

Deutschland: Übrige Lander:

$\begin{array}{ll}\text { S, Karger GmbH } & \text { S. Karger AG } \\ \text { Lörracher Str. 16a } & \text { Allschwilerstr. 10 } \\ \text { D-79115 Freiburg } & \text { Postfach } \\ \text { Telefon (07 61) } 45 & 2070 \quad \text { CH-4009 Basel } \\ \text { Telefax (0761) 4520714 Telefon (061) 3061111 } & \text { 4514 } \\ \text { Postgiro München } & \text { Telefax (061) 3061234 } \\ \text { 40080-807 E-Mail Karger@Karger.ch }\end{array}$

Anzeigen: S. Karger

Verlag für Medízin und Naturwissenschaften $\mathrm{GmbH}$

Lörracher Str. 16 a, D-79115 Freiburg

Telefon (07 61) 452070.

Gültig ist die Preisliste Nr. 9 vom 1. Januar 1996.

Für den Inhalt außerhalb des redaktionellen Teiles (insbesondere Anzeigen,

Industrieinformationen, Presse-zitate und Kongreßinformationen) übernehmen Schrift-leitung, Beirat und Verlag keine Gewähr. Eine Markenbezeichnung kann warenzeichenrechtlich geschützt sein, auch wenn bei ihrer Verwendung in dieser Zeitschrift das Zeichen ${ }^{\circledR}$ oder ein anderer Hinweis auf etwa bestehende Schutzrechte fehlen sollte. Für Satz-fehler, insbesondere bei Dosierungsangaben, wird keine Gewähr übernommen.

Die Zeitschrift sowie alle in ihr enthaltenen einzelnen Beiträge und Abbildungen sind urheberrechtlich geschützt. Jede Verwertung, die nicht ausdrücklich vom Urheberrechtsgesetz zugelassen ist, bedarf der vorherigen Zustimmung des Verlags. Das gilt insbesondere für Vervielfältigungen, Bearbeitungen, Übersetzungen, Mikro-verfillmungen und die Einspeicherung und Verarbeitung in elektronischen Systemen. Fotokopien dürfen nur für den persönlichen Gebrauch als Einzelkopien hergestellt werden. Jede im Bereich eines gewerblichen Unternehmens zulässig hergestellte oder benutzte Kopie dient gewerblichen Zwecken gem. § 54(2) UrhG und verpflich-tet zur Gebührenzahlung an die Verwertungsgesellschaft WORT, Abt. VG Wissenschaft, Goethestraße 49, D-80336 München.

(C) Copyright 1996 by S. Karger

Verlag für Medizin und Naturwissenschaften $\mathrm{GmbH}$

Lörracher Str. 16 a, D-79115 Freiburg

Verlagsleitung und presserechtlich verantwortlich:

Sibylle Hopf

Assistenz: Susanne Meister 
Herstellung: Georg Brunner

Anzeigenverwaltung: Christiane Opitz

Satz und Druck: Walter Biering GmbH Grafischer Betrieb Freisinger Landstraße 21 D-80939

München

Zusammen mit Heft 6/96 wird Supplement 1/96:

R. Osieka (Hrsg.): Resistance to Antineoplastic Agents

Is Multifaceted, ISBN 3-8055-6429-5, ausgeliefert.

$\mathrm{W}$

II

Inhalt Band 19,1996

ONKOLOCIE

Editorials

3208 Organerhaltung bei malignen Tumoren - Eine ständige Herausforderung

Schlag, P.M. (Berlin)

3209 Zahlreiche neue onkologische Lehrbücher - soeben erschienen

Queißer, W. (Mannheim); Huber, H. (Wien)

$2 \quad 176 \mathrm{pS} 2-$ Protein, EGFR und Cathepsin D in Verbindung mit

etablierten Prognosefaktoren im frühen Metastasierungs-/ Rezidivierungsgeschehen des

Mammakarzinoms

Schmidt, R., Sorger, D., Walter, E, Schönfelder, M., Preiss, R. (Leipzig)

3242 Neoadjuvante Chemotherapie ermöglicht brusterhaltendes

Operieren bei Patientinnen mit ausgedehnten primären Mammakarzinomen: Erste Ergebnisse

Taucher, S.; Gnant, M.; Djavanmard, M.; Kandioler, D,; Götzinger, P.; Rudas, M.; Steger, G.;

Jakesz, R. (Wien)

Originalarbeiten

154 Chirurgische Behandlung von Wirbelsäulenmetastasen

Rachbauer, R; Klestil, Th,; Krismer, M.; Sterzinger, W. (Innsbruck)

162 Adjuvante Chemotherapie mit 5-Fuorouracil und

Folinsäure bei kolorektalen Karzinomen: Bestimmung der Toxizität

Hartung, G.; Queißer, W.; Diezler, P.; Hagmüller, E.; Edler, L.; Jacob, I.; Wojatscheck, C;

Seifert, A.; Weiss, H.; Weh, H.-I; Hoffknecht, M.; Clemens, M.R.; Fritze, D.; Katz, R.; Härle, M.; for the Study Group Colon and Rectum Carcinoma

168 Das Selbstkonzept von Patientinnen, die unkonventionelle Heilmethoden verwenden mögliche Hinweise für eine besseres Verständnis

Münstedt, K.; Kirsch, K.; Milch, W.; Sachsse, S.; Reimer, Ch.; Vahrson, H. (Gießen)

$2 \quad 140$ Adjuvante Behandlung mit Levamisol und 5-Fluorouracil

bei Patienten nach kurativ reseziertem Kolonkarzinom im Stadium Dukes C (TNM III): Mehr

Nacheile als Vorteile

Wassner, A.; Heidemann, E. (Stuttgart)

2147 Kombinierte lokoregionale und systemische adjuvante Chemotherapie des

Rektumkarzinoms Stadium I und II

Kornek, G. V. (Wien); Depisch, D. (Wr. Neustadt); Salem, G.

(St. Pölten); Karall, M. (Wr. Neustadt); Rohrbacher, M. (St. Pölten);

Scheithauer, W. (Wien) 
2153 Phase-I-Studie zur Dosiseskalation von intravenösem Treosulfan bei therapierefraktären Karzinomen

Harstrick, A.; Wilke, H,; Eberhardt, W,; Klaassen, U.; Strumberg, D.; Korn, M.; Scheulen, M.E. (Essen); Baumgart, J. (Hamburg); Seeber, S. (Essen)

2159 Behandlung metastasierter Weichteilsarkome des

Erwachsenen mit einer Kombination aus Doxorubicin und If osfamid: Verbessert G-CSF die hämatologische Verträglichkeit?

Weh, H. I; de Wit, M.; Zornig, C; Hossfeld, D.K. (Hamburg)

2164 Plazebokontrollierte Studie von Medroxyprogesteronazetat bei gastrointestinalen

Malignomen und Kachexie

Kornek, G.V.; Schenk, T.; Ludwig, H,; Hejna, M.; Scheithauer, W (Wien)

2170 Vergleich der klinischen Wertigkeit von CEA, CA 15-3 und MCA beim Mammakarzinom

Bremer, K.; Micus, S.; Bremer, G. (Bochum); Eberhard, A. (Dortmund)

3248 Lobaplatin (D-19466) bei Patienten mit fortgeschrittenem nichtkleinzelligem

Bronchialkarzinom: Eine Untersuchung der Phase-II-Studiengruppe der Arbeitsgemeinschaft Internistische Onkologie (AIO)

Manegold, C; Drings, P. (Heidelberg); Gatzemeier, U. (Großhanddorf); Pawel, J.v.

(Gauting);Fiebig, H.H. (Freiburg); Queißer, W (Mannheim); Edler, L. (Heidelberg)

3253 Funktionelle Bildgebung mit Hilfe der Positronenemissions-

tomographie bei Patienten mit malignem Melanom

Eil, A.; Dimitrakopoulou-Strauss, A.; Tilgen, W,; Oberdorfer, F; Doll, I; Strauss, L.G.

(Heidelberg)

$4 \quad 328$ Klinische Phase-II-Studie mit Lobaplatin (D-19466)

bei vorbehandelten Patienten mit kleinzelligem Bronchialkarziom

Fiebig, H.H.; Henß, H. (Freiburg); von Pawel, I. (Gauting); Gatzemeier, U. (Großhansdorf);

Manegold, Ch.; Edler, L. (Heidelberg); Berdel, W (Berlin)

5410 Wöchentliche Hochdosis-5-Fluorouracil-24-Stunden-

Dauerinfusion kombiniert mit intermediär dosierten Folinsäurebolus bei metastasiertem

Dickdarmkarzinom

Stoffregen, C; Zurborn, K.-H; Boehme, V; Schmid, A.; Lorenz, G.; Arendt, T.; Fölsch, U.R.

(Kiel)

5416 Phase-II-Studie mit wöchentlich hochdosiertem

5-Fluorouracil und Folinsäure sowie zweiwöchentlich alternierend Cisplatin und Epirubicin

(FUFACE) bei Patienten mit fortgeschrittenem Magenkarzinom

Stahl, M,; Vanhoefer, U. (Essen); Fink, U. (München); Korn, M.; Eigler, FW. (Essen); Siewert, J.R. (München); Seeber, S.; Wilke, H. (Essen)

5419 Rezidivanalyse nach simultaner Radio-Chemotherapie inoperabler Kopf-Hals-Karzinome

Wendt, T.G; Panzer, M.; Wustrow, T.P.U.; Hartenstein, R. (München)

5424 Perioperative Veränderungen von Körperzusammen-

setzung und Stoffwechsel bei Patienten mit kolorektalem Karzinom in Abhängigkeit vom

Tumorstadium

Weimann, A.; Raab, R.; Selberg, O.; Bischoff, S.; Bornemann, K. (Hannover); Müller, M.I

(Kiel); Meyer, H.-J. (Hannover)

5430 Wirkung von Tamoxifen und Östrogen auf die Strahlen-empfindlichkeit von MCF-7-

Mammakarzinomzellen 
Böhning, K. (Göttingen); Busch, M. (München); Rave-Fränk, M. (Göttingen); Dühmke, E. (München)

\author{
$\mathrm{K} \Lambda \mathrm{R} \Gamma \mathrm{iFR} \quad \theta 1996 \mathrm{~S} \cdot \mathrm{Ka} \mathrm{T}^{7} / 8 \mathrm{er} \mathrm{GmbH}$, Freiburg \\ $\Gamma \mathrm{V} / \cdot \backslash \mathrm{IXVJ}$ L·IX $\quad$ Fax (07 61) 4520714 \\ http://www.karger.ch/journals/onk/onkdes.htm \\ III
}

6490 Phase-I- und Pharmakokinetikstudie mit LU103793

(Cemadotin Hydrochlorid) als intravenöse Bolusinfektion bei Patienten mit Malignomen

Mross, K. (Hamburg, Freiburg); Herbst, K. (Hamburg); Berdel, W.E.; Korfel. A. (Berlin); von

Broen, I.-M.; Bankmann, Y. (Ludwigshafen); Hossfeld, D.K. (Hamburg)

6496 Einsatz von Dacarbazin oder Carboplatin/VP-16 bei

fortgeschrittenen Weichteilsarkomen nach Vorbehandlung mit Dvxorubicin und Ifosfamid

Holstein, K.: Weh, H.J.; Walter, T. A.; Hossfeld, D.K. (Hamburg)

6501 Effekt der Kombination von natürlichem humanem Beta-Interferon und Bestrahlung auf humane Tumorzellen

Busch, M. (München); Rave-Fränk, M.; Franke, T. (Göttingen); Dühmke, E. (München)

Übersichtsarbeiten

16 Maligne Gliome des Gehirns und die Grenzen der Chirurgie

Steinmetz, A.; Schackert, G. (Dresden)

116 Gentherapie: Ansätze zur Behandlung maligner Gliome

Schackert, G.; Frank, St.; Schackert, H.K. (Dresden)

124 Parameter zu Tumorprogression und Metastasen bei Blasenkarzinomen

Otto, T.; Goepel, M; Krege, S.; Ruben, H. (Essen)

131 Systemische Chemotherapie des fortgeschrittenen Harnblasenkarzinoms

Bex, A.; Otto, T.; Goepel, M,; Rübben, H. (Essen)

136 Wilms-Tumor: State of the Art

Graf, N,; Weirich, A. (Homburg/Saar)

2114 Immuntherapie von Tumoren mit bispezifîschen Antikörpern

Hartmann, F; Renner, C; Pfreundschuh, M. (Homburg/Saar)

2119 Neuroendokrine Tumoren des gastroenteropankreatischen Systems: I. Aktuelle Diagnostik Scherübl, H.; Faiss, S.; Zimmer, T,; Riecken, E.-O; Wiedenmann, B. (Berlin)

2126 Biochemische Marker des Knochenumbaus

Engler, H.; Thürlimann, B.; Riesen, W.F. (St. Gallen)

2132 Wie beeinflußt die Ernährung die Entwicklung des

Kolonkarzinoms? Wechselwirkung zwischen genetischen Veränderungen und Nährstoffen bei der Deregulation des Wachstums

Marian, B. (Wien)

3211 Möglichkeiten der Organerhaltung beim

Ösophaguskarzinom

Stahl, M,; Wilke, H. (Essen)

3214 Neuroendokrine Tumoren des gastroenteropankreatischen Systems: II. Aktuelle

Therapiekonzepte

Scherübl, H,; Buhr. H.; Faiss, S.; Zimmer, T.; Riecken, E.-O.; Wiedenmann, B. (Berlin) 3221 Adjuvante Chemotherapie und Induktionschemotherapien beim nichtkleinzelligen Bronchialkarzinom 
Pirker, R.; Zöchbauer, S.; Krajnik, G. (Wien); Salzer, G.M. (Innsbruck); Eckersberger, F,; Huber, H. (Wien)

3226 Ein rationaler Ansatz zur Therapie kutaner T-Zell-Lymphome

Dummer, R.; Häffner, A. C; Hess, M,; Burg, G. (Zurich)

3234 Der lange Weg von der molekularen Basis zum klinischen

Nutzen in der Behandlung von Ewing-Tumoren

Kovar, H.; Zoubek, A.; Gadner, H. (Wien)

4290 Organsparende Resektionen bei malignen

Lungenerkrankungen

Schneider, R; Trainer, S.; Schirren, J.; Vogt-Moykopf, I. (Heidelberg)

4296 Blasenerhalt beim invasiven lokal begrenzten Blasentumor

Jung, R; Jakse, G (Aachen)

4302 Bewertung der maximalen Androgenblockade in der Therapie des fortgeschrittenen

Prostatakarzinoms

Altwein, J.E.; Silchinger, J. (München)

4308 Chemo- und Radiochemotherapie des fortgeschrittenen Pankreaskarzinoms

Mergenthaler, H.-G; Lüftner, D.; Possinger, K. (Berlin)

4315 Zytoprotektion mit Amifostin (Ethyol) in der Chemo-

und Radiotherapie: Eine neue Strategic in der Onkologie

Rieth, A.; Schuth, J.; Kudielka, R. A. (München)

4322 Prädiktive Untersuchungen für die Radiotherapie:

Die Rolle von Messungen zur Tumorproliferation (Tpot)

Terry, N.H. A. (Houston, TX)

5385 Das Mammakarzinom und das Auge. Literaturübersicht

Debois, J.M.; Haustrate, EM. (Antwerpen, Belgien)

5394 Verbesserte Möglichkeiten der Sphinktererhaltung in der operativen Therapie des

Rektumkarzinoms

Schlag, P.M. (Berlin)

5399 Organerhaltung bei Patienten im Kopf-Hals-Karzinomen

Stenson, K.M.; Vokes, E,E. (Chicago, IL)

5404 Neue Konzepte für Phase-I-Studien in Hämatologie und

Onkologie: Prinzipien und Anwendung des «Continual Reassessment Model»

Hanauske, A.-R. (München); Edler, L. (Heidelberg)

6464 Der Zellzyklus - Theorie und Anwendung in der

Tumorpathologie

Parwaresch, R.; Rudolph, P. (Kiel)

6474 Methoden zum Nachweis von MDRl/P-Glykoprotein in klinischen Tumorproben

Lehnert, M. (St. Gallen)

6480 Neue Einblicke in die Behandlung des superfiziellen Transitionalzellkarzinoms der

Harnblase

Reijke de, T.M.; Kurth, K.; Boer de, E.C.; Schamhart, DH. J. (Amsterdam)

Kurze Übersichten

144 Essentielle Thrombozythämie - Diagnostik, Komplikationen und Behandlung

Lengfelder, E.; Hochhaus, A.; Hehlmann, R. (Mannheim)

IV 
Inhalt Onkologie, Band 19,1996

149 Management of Kaposi's Sarcoma

Mitrou, PS. (Frankfurt/M.)

Clinical Information

194 Postoperative Care of Patients with Carcinomas of the

Mamma - Recommendations of a Consensus Conference

Hellriegel, K.P. (Berlin); Schulz, K.D. (Marburg)

2192 Civil Liability in Radiation Therapy - A Study of German

and Austrian Judicature

Resch-Holeczke, A.; Ofner, H.; Haverkamp, U.; Potter, R. (Wien)

3268 Population-Related Cancer Registration in Germany.

Developmental Trends of the Most Frequent Cancer Localizations

Ziegler, H.; Stegmaier, C. (Saarbrücken)

$4 \quad 354$ Regarding the Dilemma of Clinical Research in Germany

Queißer, W. (Mannheim)

4360 Consensus: Diagnostics and Treatment of Gastric Lymphoma

4364 'Brückenpflege' in Oncology: The Connecting Link between

Clinical and Home Care. Experiences at Klinikum Mannheim

Gramlich, D. (Mannheim)

5446 Resolution concerning the Development of Medical

Oncology and Hematology in Germany

Kleeberg, U.R., et al. (Hamburg)

6513 Guidelines for the Therapy of Esophageal Cancer

6516 Guidelines for the Therapy of Exocrine Pancreatic Cancer

Casuistic Contributions

3260 Cancer Presenting as Anterior Axillary Mass: Ectopic

Breast Cancer? Report of Five Cases

Möhr, T.; Queißer, W. (Mannheim)

$4 \quad 337$ Long-Term Stabilization in a Patient with Nonresected

Pancreatic Carcinoma Undergoing Chemotherapy

Jungius, K.-P; Queißer, W.; Queißer, U.; Georgi, M. (Mannheim)

5437 Treatment of Prolymphocytic Leukemia with Pentostatin

(Nipent ${ }^{\circledR}$ ): A Case Report

Wilier, A.; Käfer, G.; Queißer, W.; Hehlmann, R.; Hastka, J. (Mannheim)

5440 Hodgkin's Disease after Splenic Immunocytoma: Rare Manifestation of a Dual

Lymphoma

Ranft, K.; Reuter, H. (Penzberg)

$5 \quad 444$ Facial Angioedema Associated with Granulocyte

Colony-Stimulating Factor (G-CSF) Treatment

Laurent, D.; Schmidberger, H.; Pradier, O.; Hess, C.F. (Göttingen)

6506 Axillary Metastatic Spread in Connection with Local

Recurrence of a Renal Cell Carcinoma 22 Years after the First Diagnosis - Case Report and

Survey of the Literature

Wiegel, T.; Degner, C; Comely, D.; Runkel, N. (Berlin)

6508 13-Cis Retinoic Acid, Interferon-alpha and Concomitant

Irradiation in a Patient with Non-Small-Cell Lung Cancer -Description of Toxicity and Response 
Schiebe, M.; Hoffmann, W.; Vogel, U.; Bültmann, B.; Bamberg, M. (Tubingen)

Fax Communication

4334 Correlation between the Expression of Ki67 and

HLA-DR Antigens in Fotemustine-Treated Melanoma Cell Lines

Paul, D.R.; Gawlik, C; Versteeg, R.; Arps, H.; Kleeberg U.R. (Hamburg)

Commentaries

175 What Is the Role of Surgery in Multimodality Treatment of Localized Oesophagal Cancer?

Stahl, M. (Essen)

178 High-Dose Chemotherapy as Adjuvant Treatment for Breast Cancer - Some Statistical

Arguments

Schmoor, C, Schumacher, M. (Freiburg)

Oncological Education

181 Concepts of Neoadjuvant Therapy

Hörmann, K. (Mannheim); Manegold, C. (Heidelberg); Queißer, W.; Híeber, U. (Mannheim);

Hartung, G; Sauer, O.; Köhrmann, K.U.; Weber, A.; Siegsmund, M.; Alken, P.; Weber, A.;

Köhrmann, K.U.; Siegsmund, M.; Alken, P.; Neises, M.; Strittmatter, H. J. (Heidelberg)

2184 Randomization and Patient's Information

4340 Diagnosis and Therapy of Pituitary Diseases

Mann, K; Stolke, D. (Essen); Renner, U; Stalla, G.K. (München); Hörmann, R.; Seifert, V.

(Essen); Jockenhövel, F; Müller-Wieland, D. (Köln); Sailer, B. (Essen)

Letters to the Editors

174 Mistletoe in Oncology: Fact or Fiction?

Gabius, H.-J. (München); Gabius, S. (Rosenheim)

2181 Neumaier, M.; Gerhard, M. (Hamburg); Juhl, H. (Kiel) concerning: Panthel K, Riethmüller G: Methods for Detection of Micrometastatic Carcinoma Cells in Bone Marrow, Blood and Lymph Nodes

2182 Mistletoe in Oncology: Facts not Fiction!

Beuth, J. (Köln) concerning:

Gabius H J, Gabius S: Mistletoe in Oncology: Fact or

Fiction?

3266 Adjuvant Chemotherapy of Rectal Carcinoma

Weh, H.J. (Hamburg), concerning:

Kornek G, et al.: Combined locoregional and systemic adjuvant chemotherapy of stage II and III rectal carcinoma. Onkologie 1996;19:147-151.

3266 Hennemann, H.H. (Mannheim), concerning:

Possinger K: Tribute to Professor H. Stobbe. Onkologie 1995;18:290.

Contents Onkologie, Vol. 19,1996

IX

149 Behandlung des Kaposi-Sarkoms

Mitrou, PS. (Frankfurt/M.)

Klinische Information

194 Nachsorge von Mammakarzinom-Patientinnen -

Empfehlungen einer Konsensus-Tagung

Hellriegel, K.P. (Berlin); Schulz, K.D. (Marburg)

2192 Zivilrechtliche Haftung in der Strahlentherapie - 
Eine Untersuchung der deutschen und österreichischen Judikatur

Resch-Holeczke, A.; Ofner, H.; Haverkamp, U.; Potter, R. (Wien)

3268 Bevölkerungsbezogene Krebsregistrierung in Deutschland.

Entwicklungstrends der häufigsten Krebslokalisationen

Ziegler, H.; Stegmaier, C. (Saarbrücken)

4354 Zum Dilemma der klinischen Forschung in Deutschland

Queißer, W. (Mannheim)

4360 Konsensus der CAO, AIO und ARO zur Díagnostik und Therapie des primären

Magenlymphoms

4364 Brückenpflege in der Onkologie: Bindeglied zwischen

klinischer und häuslicher Versorgung, Erfahrungen am Mannheimer Klinikum

Gramlich, D. (Mannheim)

5446 Resolution zur Entwicklung der internistischen

Onkologie und Hämatologie in Deutschland

Kleeberg, U.R., et al. (Hamburg)

6513 Leitlinien zur Therapie des Ösophaguskarzinoms

6516 Leitlinien zur Therapie des exokrinen Pankreaskarzinoms

Kasuistiken

3260 Karzinom in der vorderen Axilla: Ektopes Mammakarzinom?

Fünf Fallbeispiele

Möhr, T.; Queißer, W. (Mannheim)

4337 Langzeit-Stabilisierung eines Patienten mit nichtreseziertem

Pankreaskarzinom unter Chemotherapie

Jungius, K.-P; Queißer, W.; Queißer, U.; Georgi, M. (Mannheim)

5437 Behandlung der Prolymphozyten-Leukämie mit Pentostatin

(Nipent ${ }^{\circledR}$ ): Eine Kasuistik

Wilier, A.; Käfer, G.; Queißer, W.; Hehlmann, R.; Hastka, J. (Mannheim)

5440 Morbus Hodgkin nach milzdominantem Immunozytom Seltene Form eines

Doppellymphoms

Ranft, K.; Reuter, H. (Penzberg)

$5 \quad 444$ Faziales Angioödem nach Behandlung mit

Granulozyten-Kolonie-stimulierendem Faktor (G-CSF)

Laurent, D.; Schmidberger, H.; Pradier, p.; Hess, C.R (Göttingen)

6506 Axilläre Metastasíerung in Verbindung mit einem

Lokalrezidiv bei einem Nierenzellkarzinom 22 Jahre nach Erstdiagnose - Kasuistik und

Literaturübersicht

Wiegel, T.; Degner, G; Comely, D.; Runkel, N. (Berlin)

6508 13-cis-Retinsäure und alpha-Interferon begleitend zur Strahlentherapie eines

nichtkleinzellingen Bronchialkarzinoms

Schiebe, M.; Hoffmann, W.; Vogel, U; Bültmann, B.; Bamberg, M. (Tubingen)

Fax-Kommunikation

4334 Korrelation zwischen der Expression des Ki67 und des HLA-DR-Antigens in Fotemustinebehandelten Melanomzell-Linien

Paul, D.R.; Gawlik, C; Versteeg, R.; Arps, H.; Kleeberg, U.R. (Hamburg)

Kommentare 
175 Welchen Stellenwert besitzt die Chirurgie im multimodalen Konzept des lokalisierten Ösophaguskarzinoms?

Stahl, M. (Essen)

178 Hochdosischemotherapie beim Mammakarzinom in der adjuvanten Situation - ein

Kommentar aus statistischer Sicht

Schmoor, G; Schumacher, M. (Freiburg)

Onkologische Fortbildung

181 Neoadjuvante Therapiekonzepte

Hörmann, K. (Mannheim); Manegold, C. (Heidelberg); Queißer, W.; Hieber, U. (Mannheim); Hartung, G; Sauer, O.; Köhrmann, K.U.; Weber, A.; Siegsmund, M.; Alken, P.; Weber, A.;

Köhrmann, K.U.; Siegsmund, M.; Alken, P.; Neises, M,; Strittmatter, H. J. (Heidelberg)

2184 Randomisation und Patientenaufklärung

4340 Diagnostik und Therapie von Hypophysenerkrankungen

Mann, K; Stolke, D. (Essen); Renner, U; Stalla, GK. (München); Hörmann, R.; Seifert, V.

(Essen); Jockenhövel, F; Müller-Wieland, D. (Köln); Sailer, B. (Essen)

Briefe an die Herausgeber

174 Mistellektin in der Onkologie: Dichtung oder Wahrheit?

Gabius, H.-J. (München); Gabius, S. (Rosenheim)

2181 Neumaier, M., Gerhard, M., Mil, H. zu:

Panthel K, Riethmüller G: Methods for Detection of Micrometastatic Carcinoma Cells in Bone Marrow, Blood and Lymph Nodes

2182 Mistellektin in der Onkologie: Tatsachen nicht Fiktion!

Beuth, J. zu:

Gabius HJ, Gabius S: Mistletoe in Oncology: Fact or

Fiction?

3266 Adjuvante Chemotherapie des Rectumkarzinoms

Weh, H. J. (Hamburg), zu:

Kornek G, et al.: Kombinierte lokoregionale und systemische adjuvante Chemotherapie des

Rektumkarzinoms Stadium II und III. Onkologie 1996;19:147-151.

3266 Hennemann, H.H. (Mannheim), zu:

Possinger K. Laudatio für Professor H. Stobbe. Onkologie 1995; 18:290

Inhalt Onkologie, Band 19,1996

$\mathrm{V}$

Kongreßberichte 1 97, 4368

B uchbesprechnungen 1 99,2 198, 3 278, 4 371, 5 450, 6520

Mitteilungen onkologischer Gesellschaften 1103,2 201, 3 282, 4 374, 5 455, 6526

Industrieforum 1101, 2 200, 3 280, 4 372, 5 452, 6525

Nachruf

6524

Supplement 1

Resistance to Antineoplastic Agents Is Multifaceted

3rd International Meeting on Drug Resistance Editor: R. Osieka, Aachen

VI

Inhalt Onkologie, Band 19,1996 
Contents Vol.

ONKOLOCIE

19,1996

Editorials

3208 Organ Preservation in Malignant Tumors - The Continuing Challenge

Schlag, P.M. (Berlin)

3209 Numerous New Oncological Textbooks Recently Published

Queißer, W. (Mannheim); Huber, H. (Wien)

Original Articles

154 Surgical Treatment of Metastatic Disease of the Spine

Rachbauer, R.; Klestil, Th.; Krismer, M.; Sterzinger, W. (Innsbruck)

162 Adjuvant Chemotherapy with 5-Fluorouracil and Folinic Acid in Colorectal Cancer:

Evaluation of Toxicity

Hartung, G.; Queißer, W.; Diezler, R; Hagmüller, E.; Edler, L.; Jacob, I.; Wojatscheck, C;

Seifert, A.; Weiss, H.; Wen, H.-I; Hoffknecht, M.; Clemens, M.R.; Fritze, D.; Katz, R,; Härle, M.; for the Study Group Colon and Rectum Carcinoma

168 Self-Concept of Female Patients Using Unconventional Cancer Therapy - Possible

Implications for Better Understanding

Münstedt, K.; Kirsch, K.; Milch, W.; Sachsse, S.; Reimer, Ch.; Vahrson, H. (Gießen)

2140 Levamisole and 5-Fluorouracil as an Adjuvant Therapy

for Patients after Curative Resection of Colon Carcinoma Dukes' Stage C (TNM III): More

Disadjuvantages than Advantages

Wässner, A.; Heidemann, E. (Stuttgart)

2147 Combined Locoregional and Systemic Adjuvant

Chemotherapy of Stage II and III Rectal Carcinoma

Kornek, G V. (Wien); Depisch, D. (Wr. Neustadt); Salem, G.

(St. Pölten); Karall, M. (Wr. Neustadt); Rohrbacher, M. (St. Pölten);

Scheithauer, W. (Wien)

2153 A Phase I Dose Escalation Trial of Intravenous Treosulfan in Refractory Cancer

Harstrick, A.; Wilke, H.; Eberhardt, W; Klaassen, U.; Strumberg, D.; Korn, M.; Scheulen, M.E.

(Essen); Baumgart, J. (Hamburg); Seeber, S. (Essen)

2159 Treatment of Adult Metastatic Soft-Tissue Sarcoma with Doxorubicin/Ifosfamide: Better

Hematologic Tolerance by G-CSF?

Weh, H.J.; de Wit, M.; Zornig, C; Hossfeld, D.K. (Hamburg)

2164 Placebo-Controlled Trial of Medroxyprogesterone

Acetate in Gastrointestinal Malignancies and Cachexia

Kornek, G.V.; Schenk, T.; Ludwig, H,; Hejna, M.; Scheithauer, W. (Wien)

2170 Comparison of the Clinical Relevance of CEA, CA 15-3 and MCA in Breast Cancer

Bremer, K.; Micus, S.; Bremer, G. (Bochum); Eberhard, A. (Dortmund)

$2176 \mathrm{pS} 2$ Protein, EGFR and Cathepsin D in Association with

Established Prognostic Factors in Early Recurrence of Breast Cancer

Schmidt, R., Sorger, D., Walter, E, Schönfelder, M., Preiss, R. (Leipzig)

3242 Neoadjuvant Chemotherapy Enables Breast-Conserving

Surgery in Patients with Very Large Breast Cancers: Preliminary Results 
Taucher, S.; Gnant, M.; Djavanmard, M.; Kandioler, D.; Götzinger, P.; Rudas, M.; Steger, G; Jakesz, R. (Wien) 3248 Lobaplatin (D-19466) in Patients with Advanced

Non-Small-Cell Lung Cancer: A Trial of the Association for Medical Oncology (AIO) Phase II Study Group

Manegold, C.;Drings, P. (Heidelberg); Gatzemeier,Ul (Großhanddorf); Pawel, J.v. (Gauting); Fiebig, H. H. (Freiburg); Queißer, W (Mannheim); Edler, L. (Heidelberg)

3253 Functional Imaging with Positron Emission Tomography in Patients with Malignant Melanoma

Eil, A.; Dimitrakopoulou-Strauss, A.; Tilgen, W.; Oberdorfer, F; Doll, I; Strauss, L.G

(Heidelberg)

4328 Phase II Clinical Trial of Lobaplatin (D-19466) in

Pretreated Patients with Small-Cell Lung Cancer

Fiebig, H.H.; Henß, H. (Freiburg); von Pawel, I. (Gauting); Gatzemeier, U. (Großhansdorf); Manegold, Ch.; Edler, L. (Heidelberg); Berdel, W (Berlin)

5410 Weekly High-Dose 5-Fluorouracil 24-Hour Infusion

and Intermediate-Dose Folinic Acid Bolus in Metastatic Colorectal Cancer

Stoffregen, G; Zurborn, K.-H.; Boehme, V.; Schmid, A.; Lorenz, G; Arendt, T.; Fölsch, U.R.

(Kiel)

5416 Phase II Study of Weekly High-Dose 5-Fluorouracil and Folinic Acid plus Biweekly

Alternating Cisplatin and Epirubicin (FUFACE) in Patients with Advanced Gastric Carcinoma

Stahl, M.; Vanhoefer, U. (Essen); Fink, U. (München); Korn, M.; Eigler, F. W (Essen); Siewert, J.R. (München); Seeber, S.; Wilke, H. (Essen)

5419 Pattern of Failure in Long-Term Survivors after Radio-Chemotherapy for Inoperable Head and Neck Cancer

Wendt, T.G.; Panzer, M.; Wustrow T.P.U.; Hartenstein, R. (München)

5424 Perioperative Changes in Body Composition and

Metabolism in Patients with Colorectal Cancer according to Tumor Stage

Weimann, A.; Raab, R.; Selberg, O.; Bischoff, S.; Bornemann, K. (Hannover); Müller, M.J.

(Kiel); Meyer, H.-J. (Hannover)

5430 Effect of Tamoxifen and Estrogen Treatment on the Radiosensitivity of MCF-7 Breast

Cancer Cells

Böhning, K. (Göttingen); Busch, M. (München); Rave-Fränk, M. (Göttingen); Dühmke, E. (München)

http://www.karger.ch/joumals/onk/onkdes.htm

VII

6490 Phase I Clinical and Pharmacokinetic Study of LU103793 (Cemadotin Hydrochloride) as an Intravenous Bolus Injection in Patients with Metastatic Solid Tumors

Mross, K. (Hamburg, Freiburg); Herbst, K. (Hamburg); Berdel, W.E.; Korfel, A. (Berlin); von Broen, I.-M.; Bankmann, Y. (Ludwigshafen); Hossfeld, D.K. (Hamburg)

6496 Salvage Chemotherapy with Dacarbazine or

Carboplatin/VP-16 of Advanced Soft Tissue Sarcoma Pretreated with Doxorubicin/Ifosfamide

Holstein, K.; Weh, H.J.; Walter, T. A.; Hossfeld, D.K. (Hamburg)

6501 Effect of Combined Natural Human Beta-Interferon and Radiation on Human Tumor Cells Busch, M. (München); Rave-Fränk, M.; Franke, T. (Göttingen); Dühmke, E. (München) 
Review Articles

16 Malignant Gliomas of the Brain and Surgical Limitations

Steinmetz, A.; Schackert, G. (Dresden)

116 Gene Therapy: Approaches for the Treatment of Malignant Gliomas

Schackert, G.; Frank, St.; Schackert, H.K. (Dresden)

124 Parameters of Tumor Progression and Metastases in Bladder Carcinomas

Otto, T.; Goepel, M,; Krege, S.; Rübben, H. (Essen)

131 Systemic Chemotherapy for Advanced Bladder Carcinoma

Bex, A.; Otto, T.; Goepel, M; Rübben, H. (Essen)

136 Wilm's Tumour - the State of the Art

Graf, N.; Weirich, A. (Homburg/Saar)

2114 Tumor Immunotherapy with Bispecific Antibodies

Hartmann, E; Renner, C; Pfreundschuh, M. (Homburg/Saar)

2119 Neuroendocrine Tumors of the Gastroenteropancreatic System: I. Diagnostic Advances

Scherübl, H.; Faiss, S.; Zimmer, T.; Riecken, E.-O; Wiedenmann B. (Berlin)

2126 Biochemical Markers of Bone Remodelling

Engler, H.; Thürlimann, B.; Riesen, W.F. (St. Gallen)

2132 How Does Diet Contribute to Colon Cancer Development?

Interaction between Genetic Alternations and Nutrients in the Deregulation of Growth

Marian, B. (Wien)

3211 Possibilities of Non-Resective Treatment Procedures

in Oesophageal Cancer

Stahl, M.; Wilke, H. (Essen)

3214 Neuroendocrine Tumors of the Gastroenteropancreatic System: II. Therapeutic Advances

Scherübl, H.; Buhr, H.; Faiss, S,; Zimmer, T.; Riecken, E.-O.; Wiedenmann, B. (Berlin)

3221 Adjuvant and Induction Chemotherapies in Non-Small-Cell Lung Cancer

Pïrker, R,; Zöchbauer, S.; Krajnik, G. (Wien); Salzer, G.M. (Innsbruck); Eckersberger, F; Huber, H. (Wien)

3226 A Rational Approach to the Therapy of Cutaneous T-Cell Lymphomas

Dummer, R.; Häffner, A.C.; Hess, M,; Burg, G. (Zurich)

3234 A Long Way from Definition of the Molecular Basis to

Benefit in the Clinical Management of Ewing Tumours

Kovar, H.; Zoubek, A.; Gadner, H. (Wien)

4290 Organ-Preserving Resection Methods on Lung Tumors

Schneider, R; Trainer, S.; Schirren, I; Vogt-Moykopf, I. (Heidelberg)

4296 Bladder Preservation in Invasive Locally Confined Bladder Cancer

Jung, R; Jakse, G (Aachen)

4302 An Assessment of Maximal Androgen Blockade in the Treatment of Advanced Prostatic

Carcinoma

Altwein, IE.; Silchinger, I (München)

4308 Chemotherapy and Chemo-Radiotherapy of Advanced Pancreatic Carcinoma

Mergenthaler, H.-G; Lüftner, D.; Possinger, K. (Berlin)

4315 Cytoprotection in Chemo- and Radiotherapy with Amifostine (Ethyol): A New Strategy in Oncology

Rieth, A.; Schuth, I; Kudielka, R. A. (München)

4322 Predictive Assays for Radiotherapy: The Role of Tumor 
Proliferation (Tpot) Measurements

Terry, N. H. A. (Houston, TX)

5385 Breast Cancer and the Eye. A Literature Review

Debois, J.M.; Haustrate, FM. (Antwerp, Belgium)

5394 Advances for Sphincter Preservation in the Surgical Therapy of Rectal Cancer

Schlag, P.M. (Berlin)

5399 Conservation Therapy in Patients with Head and Neck Carcinoma

Stenson, K.M.; Vokes, E.E. (Chicago, IL)

5404 New Clinical Trial Designs for Phase I Studies in

Hematology and Oncology: Principles and Practice of the Continual Reassessment Model

Hanauske, A.-R. (München); Edler, L. (Heidelberg)

$6 \quad 464$ The Cell Cycle - Theory and Application to Cancer

Parwaresch, R.; Rudolph, P. (Kiel)

6474 Methods to Detect MDRl/P-Glycoprotein in Clinical Tumor Samples

Lehnert, M. (St. Gallen)

6480 Current Insights in the Treatment of Superficial Transitional Cell Carcinoma of the

Bladder

Reijke de, T.M.; Kurth, K; Boer de, E.C.; Schamhart, DH. J. (Amsterdam)

Short Reviews

144 Essential Thrombocythemia: Current Opinion in Diagnosis, Clinical Course, and Treatment Lengfelder, E.; Hochhaus, A.; Hehlmann, R. (Mannheim)

VIII

Contents Onkologie, Vol. 19,1996

Congress Reports 197, 4368

Book Reviews 1 99,2 198,3 278, 4 371, 5 450, 6520

Reports of Oncological Societies 1103, 2 201, 3 282, 4 374, 5 455, 6526

Industrial Forum 1101,2 200, 3 280, 4 372, 5 452, 6525

Obituary

6524

Supplement 1

Resistance to Antineoplastic Agents Is Multifaceted

3rd International Meeting on Drug Resistance Editor: R. Osieka, Aachen

$\mathrm{X}$

Contents Onkologie, Vol. 19,1996 